\title{
ON THE COMMUTATOR SUBGROUP OF A RIGHT-ANGLED ARTIN GROUP
}

\author{
TARAS PANOV AND YAKOV VERYOVKIN
}

\begin{abstract}
We use polyhedral product models to analyse the structure of the commutator subgroup of a right-angled Artin group. In particular, we provide a minimal set of generators for the commutator subgroup, consisting of special iterated commutators of canonical generators.
\end{abstract}

\section{INTRODUCTION}

A right-angled Artin group, also known as a graph group or a partially commutative group, has $m$ generators $g_{1}, \ldots, g_{m}$ and commutativity relations $g_{i} g_{j}=g_{j} g_{i}$ for some pairs $\{i, j\}$. Such a group interpolates between a free group of rank $m$ (in which no pair of generators commutes), and a free abelian group of rank $m$ (in which every pair of generators commutes). A right-angled Artin group is therefore defined by a graph $\Gamma$ with $m$ vertices, where two vertices are joined by an edge whenever the two corresponding generators commute. Right-angled Artin groups are classical objects in geometric group theory.

Right-angled Artin groups are particular cases of graph product groups, corresponding to a sequence of $m$ groups $\boldsymbol{G}=\left(G_{1}, \ldots, G_{m}\right)$ and a graph $\Gamma$ on $m$ vertices. The graph product group $\boldsymbol{G}^{\Gamma}$ consists of words with letters from $G_{1}, \ldots, G_{m}$ in which the elements of $G_{i}$ and $G_{j}$ with $i \neq j$ commute whenever $\{i, j\}$ is an edge of $\Gamma$. The graph product group $G^{\Gamma}$ sits between the free product $G_{1} \star \cdots \star G_{m}$ (corresponding to a graph consisting of $m$ disjoint vertices) and the cartesian product $G_{1} \times \cdots \times G_{m}$ (corresponding to a complete graph). A right-angled Artin group is a graph product in which all $G_{i}$ are infinite cyclic groups $\mathbb{Z}$.

To each graph $\Gamma$ without loops and double edges one can assign its clique complex $\mathcal{K}$, which is the simplicial complex on the vertex set of $\Gamma$ whose simplices are cliques (complete subgraphs) of $\Gamma$. The simplicial complex $\mathcal{K}$ is flag, and its one-skeleton is the graph $\Gamma$. Many properties of graph products are formulated more explicitly in terms of the simplicial complex $\mathcal{K}$. We use the notation $G^{\mathcal{K}}$ for a graph product, alongside with $G^{\Gamma}$.

A graph product group $G^{\mathcal{K}}$ can be defined as the colimit of a diagram of groups over the face category of $\mathcal{K}[\mathrm{PV}]$. There is a similar colimit construction of topological spaces, known as the polyhedral product, which is fundamental in toric topology. The polyhedral product functor assigns a space $(\boldsymbol{X}, \boldsymbol{A})^{\mathcal{K}}$ to a sequence of $m$ pairs of spaces $(\boldsymbol{X}, \boldsymbol{A})=\left\{\left(X_{1}, A_{1}\right), \ldots,\left(X_{m}, A_{m}\right)\right\}$ and a simplicial complex $\mathcal{K}$ on $m$ vertices [BP1, BBCG], BP2. From the homotopy-theoretical point of view, polyhedral products $(\boldsymbol{X}, \boldsymbol{A})^{\mathcal{K}}$ have much richer structure than graph product groups $G^{\mathcal{K}}$, as the former may be used to study all sorts of higher homotopy phenomena. However, when the underlying simplicial comlex $\mathcal{K}$ is flag, the classifying space

2010 Mathematics Subject Classification. 20F65, 20F12, 57M07.

Key words and phrases. Right-angled Artin group, commutator subgroup, polyhedral product.

The research of both authors was carried out at the Steklov Institute of Mathematics and supported by the Russian Science Foundation grant no. 14-11-00414. The second author is a laureate of the Young Mathematics of Russia 2016 award. 
functor $B$ takes the graph product $(\boldsymbol{G})^{\mathcal{K}}$ to the polyhedral product $(B \boldsymbol{G})^{\mathcal{K}}$, and the polyhedral product $(E \boldsymbol{G}, \boldsymbol{G})^{\mathcal{K}}$ is the classifying space for the commutator subgroup $\left(\boldsymbol{G}^{\mathcal{K}}\right)^{\prime}$ when all $G_{i}$ are abelian. In the case of a right-angled Artin group, each classifying space $B G_{i}=B \mathbb{Z}$ is a circle, and we obtain as $(B \boldsymbol{G})^{\mathcal{K}}$ the subcomplex $\left(S^{1}\right)^{\mathcal{K}}$ in the $m$-torus, introduced by Kim and Roush in [KR].

In this paper we use the construction of polyhedral products and other techniques of toric topology to study the commutator subgroups of right-angled Artin groups and more general graph products. Apart from a purely algebraic interest, our motivation lies in the fact that these commutators subgroups arise as the fundamental groups of some remarkable aspherical spaces. We refer to [PV], BEMPP] for the discussion of topological aspects of the theory of right-angled Artin groups and their commutator subgroups.

In Section 2 we include some basic information about right-angled Artin groups and polyhedral products. In Section 3 we construct a special basis for the commutator subgroup of a free group, which is also a right-angled Artin group corresponding to $m$ disjoint vertices. Several other constructions of bases in the commutator subgroup of a free group are known, see e. g. [BK, Appendix 1] and [HW]. In Section 4 we give an explicit minimal set of generators for the commutator subgroup of an arbitrary right-angled Artin group $R A_{\mathcal{K}}$. The generators are nested iterated commutators of powers of the canonical generators of $R A_{\mathcal{K}}$ appearing in a special order prescribed by the combinatorics of $\mathcal{K}$. In Section $[5$ we generalise the result to arbitrary graph products.

We thank the anonymous referee for the most helpful comments and suggestions.

\section{Preliminaries}

Let $\mathcal{K}$ be an abstract simplicial complex on the set $[m]=\{1,2, \ldots, m\}$. We refer to a subset $I=\left\{i_{1}, \ldots, i_{k}\right\} \in \mathcal{K}$ as a simplex (or face) of $\mathcal{K}$. We assume that $\mathcal{K}$ contains the empty set $\varnothing$ and all one-element subsets $\{i\} \in[\mathrm{m}]$; the latter are referred to as the vertices of $\mathcal{K}$.

We denote by $F_{m}$ or $F\left(g_{1}, \ldots, g_{m}\right)$ a free group of rank $m$ with generators $g_{1}, \ldots, g_{m}$.

Definition 2.1. The right-angled Artin group $R A_{\mathcal{K}}$ corresponding to $\mathcal{K}$ is defined by generators and relations as follows:

$$
R A_{\mathcal{K}}=F\left(g_{1}, \ldots, g_{m}\right) /\left(g_{i} g_{j}=g_{j} g_{i} \text { for }\{i, j\} \in \mathcal{K}\right) .
$$

Clearly, the group $R A_{\mathcal{K}}$ depends only on the 1 -skeleton of $\mathcal{K}$, the graph $\mathcal{K}^{1}$.

Definition 2.2. Let

$$
(\boldsymbol{X}, \boldsymbol{A})=\left\{\left(X_{1}, A_{1}\right), \ldots,\left(X_{m}, A_{m}\right)\right\}
$$

be a sequence of $m$ pairs of pointed topological spaces, $p t \in A_{i} \subset X_{i}$, where $p t$ denotes the basepoint. For each subset $I \subset[m]$ we set

$$
(\boldsymbol{X}, \boldsymbol{A})^{I}=\left\{\left(x_{1}, \ldots, x_{m}\right) \in \prod_{k=1}^{m} X_{k}: x_{k} \in A_{k} \quad \text { for } k \notin I\right\}
$$

For a simplicial complex $\mathcal{K}$ on $[m]$, the polyhedral product of $(\boldsymbol{X}, \boldsymbol{A})$ corresponding to $\mathcal{K}$ is

$$
(\boldsymbol{X}, \boldsymbol{A})^{\mathcal{K}}=\bigcup_{I \in \mathcal{K}}(\boldsymbol{X}, \boldsymbol{A})^{I}=\bigcup_{I \in \mathcal{K}}\left(\prod_{i \in I} X_{i} \times \prod_{i \notin I} A_{i}\right),
$$

where the union is taken inside the Cartesian product $\prod_{k=1}^{m} X_{k}$.

In the case when all pairs $\left(X_{i}, A_{i}\right)$ are the same, i. e. $X_{i}=X$ and $A_{i}=A$ for $i=1, \ldots, m$, we use the notation $(X, A)^{\mathcal{K}}$ for $(\boldsymbol{X}, \boldsymbol{A})^{\mathcal{K}}$. Also, if each $A_{i}=p t$, then we use the abbreviated notation $\boldsymbol{X}^{\mathcal{K}}$ for $(\boldsymbol{X}, p t)^{\mathcal{K}}$, and $X^{\mathcal{K}}$ for $(X, p t)^{\mathcal{K}}$. 


\section{Example 2.3.}

1. Let $(X, A)=\left(S^{1}, p t\right)$, where $S^{1}$ is a circle. The polyhedral product

$$
\left(S^{1}\right)^{\mathcal{K}}=\bigcup_{I \in \mathcal{K}}\left(S^{1}\right)^{I}
$$

is a subcomplex of the $m$-torus $\left(S^{1}\right)^{m}$, sitting between the $m$-fold wedge $\left(S^{1}\right)^{\vee m}$ and the $m$-fold Cartesian product $\left(S^{1}\right)^{m}$.

2. Let $(X, A)=(\mathbb{R}, \mathbb{Z})$, where $\mathbb{Z}$ is the set of integer points on the real line $\mathbb{R}$. We denote the corresponding polyhedral product by $\mathcal{L}_{\mathcal{K}}$ :

$$
\mathcal{L}_{\mathcal{K}}=(\mathbb{R}, \mathbb{Z})^{\mathcal{K}}=\bigcup_{I \in \mathcal{K}}(\mathbb{R}, \mathbb{Z})^{I} \subset \mathbb{R}^{m}
$$

When $\mathcal{K}$ consists of $m$ disjoint points, $\mathcal{L}_{\mathcal{K}}$ is a grid in $m$-dimensional space $\mathbb{R}^{m}$ consisting of all lines parallel to one of the coordinate axis and passing though integer points. When $\mathcal{K}=\partial \Delta^{m-1}$, the complex $\mathcal{L}_{\mathcal{K}}$ is the union of all integer hyperplanes parallel to the coordinate hyperplanes.

A missing face (or a minimal non-face) of $\mathcal{K}$ is a subset $I \subset[m]$ such that $I$ is not a simplex of $\mathcal{K}$, but every proper subset of $I$ is a simplex of $\mathcal{K}$. A simplicial complex $\mathcal{K}$ is called a flag complex if each of its missing faces consists of two vertices. Equivalently, $\mathcal{K}$ is flag if any set of vertices of $\mathcal{K}$ which are pairwise connected by edges is a simplex.

A clique (or a complete subgraph) of a graph $\Gamma$ is a subset $I$ of vertices such that every two vertices in $I$ are connected by an edge. Each flag complex $\mathcal{K}$ is the clique complex of its one-skeleton $\Gamma=\mathcal{K}^{1}$, that is, the simplicial complex formed by filling in each clique of $\Gamma$ by a face.

A path-connected space $X$ is aspherical if $\pi_{i}(X)=0$ for $i \geqslant 2$. An aspherical space $X$ is an Eilenberg-MacLane space $K(\pi, 1)$ with $\pi=\pi_{1}(X)$, and also the classifying space $B \pi$ for the group $\pi$.

By the result of Kim and Roush $\left[\mathrm{KR}\right.$, Theorem 10], the polyhedral product $\left(S^{1}\right)^{\mathcal{K}}$ corresponding to a flag complex $\mathcal{K}$ is the classifying space for the right-angled Artin group $R A_{\mathcal{K}}$. The polyhedral product $\mathcal{L}_{\mathcal{K}}$ is the classifying space for the commutator subgroup $R A_{\mathcal{K}}^{\prime}$. More precisely, we have the following result.

Theorem 2.4 (see [PV] Corollary 3.3]). Let $\mathcal{K}$ be a simplicial complex on $m$ vertices, and let $R A_{\mathcal{K}}$ be the corresponding right-angled Artin group.

(a) $\pi_{1}\left(\left(S^{1}\right)^{\mathcal{K}}\right) \cong R A_{\mathcal{K}}$.

(b) $\pi_{1}\left(\mathcal{L}_{\mathcal{K}}\right) \cong R A_{\mathcal{K}}^{\prime}$.

(c) Each of the spaces $\left(S^{1}\right)^{\mathcal{K}}$ and $\mathcal{L}_{\mathcal{K}}$ is aspherical if and only if $\mathcal{K}$ is a flag complex.

Given a subset $J \subset[m]$, consider the restriction of $\mathcal{K}$ to $J$ :

$$
\mathcal{K}_{J}=\{I \in \mathcal{K}: I \subset J\},
$$

which is also known as a full subcomplex of $\mathcal{K}$.

Recall that the smash product of two pointed space $X$ and $Y$ is defined as $X \wedge Y=X \times Y /(X \times p t \cup p t \times Y)$, that is, the quotient of the product $X \times Y$ by the wedge $X \vee Y$. Given a sequence of spaces $\boldsymbol{A}=\left(A_{1}, \ldots, A_{m}\right)$ and a subset $J=\left\{j_{1}, \ldots, j_{k}\right\} \subset[m]$, we denote the smash product $A_{j_{1}} \wedge \cdots \wedge A_{j_{k}}$ by $\boldsymbol{A}^{\wedge J}$.

A theorem of Bahri, Bendersky, Cohen and Gitler [BBCG, Theorem 2.21] describes the homotopy type of the suspension of a polyhedral product $(\boldsymbol{X}, \boldsymbol{A})^{\mathcal{K}}$ in the case when all $X_{i}$ are contractible. It implies the following result. 
Theorem 2.5 ([BBCG $)$. Let $\mathcal{K}$ be a simplicial complex on $[m]$, and let $(\boldsymbol{X}, \boldsymbol{A})$ be a sequence of pairs of pointed cell complexes, in which all $X_{i}$ are contractible. Then there is the following isomorphism of integer homology groups:

$$
H_{p}\left((\boldsymbol{X}, \boldsymbol{A})^{\mathcal{K}}\right) \cong \widetilde{H}_{p-1}\left(\bigvee_{J \subset[m]}\left|\mathcal{K}_{J}\right| \wedge \boldsymbol{A}^{\wedge J}\right)
$$

\section{A BASIS FOR THE COMMUtATOR SUbGRoup OF A FREE GROUP $F_{m}$}

When $\mathcal{K}$ consists of $m$ disjoint points, the right-angled Artin group $R A_{\mathcal{K}}$ is a free group $F_{m}=F\left(g_{1}, \ldots, g_{m}\right)$. Here we construct a special basis for the commutator subgroup $F_{m}^{\prime}$.

Let $(g, h)=g^{-1} h^{-1} g h$ denote the group commutator of two elements $g, h$.

Theorem 3.1. The commutator subgroup $F_{m}^{\prime}$ of a free group $F_{m}=F\left(g_{1}, \ldots, g_{m}\right)$ is a free group freely generated by nested iterated commutators of the form

$$
\left(g_{j}^{n_{j}}, g_{i}^{n_{i}}\right),\left(g_{k_{1}}^{n_{k_{1}}},\left(g_{j}^{n_{j}}, g_{i}^{n_{i}}\right)\right), \ldots,\left(g_{k_{1}}^{n_{k_{1}}},\left(g_{k_{2}}^{n_{k_{2}}}, \ldots,\left(g_{k_{m-2}}^{n_{k_{m}-2}},\left(g_{j}^{n_{j}}, g_{i}^{n_{i}}\right)\right) \ldots\right)\right),
$$

where $n_{k} \in \mathbb{Z} \backslash\{0\}, k_{1}<k_{2}<\cdots<k_{m-2}<j>i$ and $k_{s} \neq i$ for any $s$.

Proof. Consider the covering of the wedge of circles $B=\left(S^{1}\right)^{\vee m}$ corresponding to the commutator subgroup of $\pi_{1}(B)=F_{m}$. The total space of this covering can be described as $E=E_{m}=\bigcup_{i=1}^{m} L_{i} \subset \mathbb{R}^{m}$, were $L_{i}$ is the union of all lines in $\mathbb{R}^{m}$ which are parallel to the $i$ th coordinate axis and pass through integer points. We have $E_{m}=(\mathbb{R}, \mathbb{Z})^{\mathcal{K}}$, where $\mathcal{K}$ is a simplicial complex consisting of $m$ disjoint vertices, see (2.2). The covering map $E \rightarrow B$ takes all points from the integer lattice $\mathbb{Z}^{m} \subset E$ to the basepoint of the wedge, and takes all segments of the $i$ th coordinate direction in the graph $E$ to the $i$ th circle of the wedge $B$. The induced homomorphism $\pi_{1}(E) \rightarrow \pi_{1}(B)$ is identified with the inclusion of the commutator subgroup $F_{m}^{\prime} \hookrightarrow F_{m}$.

Case $m=2$. Let $F_{2}=F\left(g_{1}, g_{2}\right)$. We need to check that the elements $\left(g_{2}^{n_{2}}, g_{1}^{n_{1}}\right)$ with $n_{k} \in \mathbb{Z} \backslash\{0\}$ form a basis of $F_{2}^{\prime}$. A word in $F_{2}$ belongs to $F_{2}^{\prime}$ if and only if the sum of the exponents of all $g_{1}$ is zero and the sum of the exponents of all $g_{2}$ is zero. Consider a reduced word $\varphi$ from $F_{2}^{\prime}$. We need to write $\varphi$ as a product of commutators $\left(g_{2}^{n_{2}}, g_{1}^{n_{1}}\right)$. To do this, we shall split off commutators of this form one by one from the left. Denote by $|\varphi|$ the number of alterations of $g_{1}$ and $g_{2}$ in the word $\varphi$. Note that if $|\varphi| \leqslant 2$, then $\varphi=1$, as $\varphi \in F_{2}^{\prime}$. We induct on the number $|\varphi|$. Assume that $\varphi=g_{1}^{i} g_{2}^{j} g_{1} \psi$ (the case $\varphi=g_{2}^{i} g_{1}^{j} g_{2} \psi$ is considered similarly). We rewrite the word as follows: $\varphi=g_{1}^{i} g_{2}^{j} g_{1}^{-i} g_{2}^{-j} g_{2}^{j} g_{1}^{i} g_{1} \psi=\left(g_{2}^{-j}, g_{1}^{-i}\right)^{-1} g_{2}^{j} g_{1}^{i+1} \psi$. Now $\varphi$ is the product of a commutator of the required form and the word $\varphi_{1}=g_{2}^{j} g_{1}^{i+1} \psi$, where $\left|\varphi_{1}\right|<|\varphi|$. Therefore, after finitely many iterations we arrive at a word $\varphi_{k}$ with $\left|\varphi_{k}\right| \leqslant 2$, i. e. $\varphi_{k}=1$.

Case $m=3$. Let $F_{3}=F\left(g_{1}, g_{2}, g_{3}\right)$. We need to check that the elements

$$
\left(g_{3}^{n_{3}}, g_{1}^{n_{1}}\right), \quad\left(g_{3}^{n_{3}}, g_{2}^{n_{2}}\right), \quad\left(g_{2}^{n_{2}}, g_{1}^{n_{1}}\right), \quad\left(g_{1}^{n_{1}},\left(g_{3}^{n_{3}}, g_{2}^{n_{2}}\right)\right), \quad\left(g_{2}^{n_{2}},\left(g_{3}^{n_{3}}, g_{1}^{n_{1}}\right)\right)
$$

with $n_{k} \in \mathbb{Z} \backslash\{0\}$ form a basis of $F_{3}^{\prime}$.

Let $\left(y_{1}, y_{2}, y_{3}\right)$ be Cartesian coordinates in $\mathbb{R}^{3}$. We contract the maximal tree in $E$ given by the union of all lines of the form

$$
\left\{y_{1}=C_{1}, y_{2}=C_{2}\right\}, \quad\left\{y_{2}=C_{2}, y_{3}=0\right\}, \quad\left\{y_{1}=y_{3}=0\right\}
$$

where $C_{1}, C_{2} \in \mathbb{Z}$ are arbitrary integer constants. Denote by $\widehat{E}$ the space obtained as the result of contraction. Then $\widehat{E}$ is a wedge of circles, and the circles in this wedge correspond bijectively to those edges of the graph $E$ that do not belong to the contracted maximal tree. These edges of $E$ are segments of the form

$$
\left[\left(c_{1}, c_{2}, c_{3}\right),\left(c_{1}+1, c_{2}, c_{3}\right)\right] \quad \text { or } \quad\left[\left(c_{1}, c_{2}, c_{3}\right),\left(c_{1}, c_{2}+1, c_{3}\right)\right],
$$


where $c_{i} \in \mathbb{Z}$ (we also have $c_{3} \neq 0$ in the former case, but this does not affect the subsequent argument). Every such segment can be completed to a loop in $E$ by adding edges from the contracted maximal tree, and we need to express the word corresponding to this loop via the commutators (3.2).

We first consider a segment $\left[\left(c_{1}, c_{2}, c_{3}\right),\left(c_{1}+1, c_{2}, c_{3}\right)\right]$. It corresponds to a circle in the wedge $\widehat{E}$ and to a loop in $E$, all of whose segments except $\left[\left(c_{1}, c_{2}, c_{3}\right),\left(c_{1}+1, c_{2}, c_{3}\right)\right]$ lying in the contracted maximal tree. This loop is given by the word $g_{2}^{c_{2}} g_{1}^{c_{1}} g_{3}^{c_{3}} g_{1} g_{3}^{-c_{3}} g_{1}^{-c_{1}-1} g_{2}^{-c_{2}}$, and can be expressed via the commutators (3.2) as follows:

$$
\begin{aligned}
& g_{2}^{c_{2}} g_{1}^{c_{1}} g_{3}^{c_{3}} g_{1} g_{3}^{-c_{3}} g_{1}^{-c_{1}-1} g_{2}^{-c_{2}} \\
& \quad=\left(g_{2}^{-c_{2}},\left(g_{3}^{-c_{3}}, g_{1}^{-c_{1}}\right)\right)\left(g_{3}^{-c_{3}}, g_{1}^{-c_{1}}\right)^{-1}\left(g_{3}^{-c_{3}}, g_{1}^{-c_{1}-1}\right)\left(g_{2}^{-c_{2}},\left(g_{3}^{-c_{3}}, g_{1}^{-c_{1}-1}\right)\right)^{-1} .
\end{aligned}
$$

Now consider a segment $\left[\left(c_{1}, c_{2}, c_{3}\right),\left(c_{1}, c_{2}+1, c_{3}\right)\right]$. It corresponds to the loop in $E$ given by the word $g_{2}^{c_{2}} g_{1}^{c_{1}} g_{3}^{c_{3}} g_{2} g_{3}^{-c_{3}} g_{1}^{-c_{1}} g_{2}^{-c_{2}-1}$, and can be expressed via the commutators (3.2) as follows:

$$
\begin{aligned}
g_{2}^{c_{2}} g_{1}^{c_{1}} g_{3}^{c_{3}} g_{2} g_{3}^{-c_{3}} g_{1}^{-c_{1}} g_{2}^{-c_{2}-1} & \\
= & \left(g_{2}^{-c_{2}}, g_{1}^{-c_{1}}\right)\left(g_{1}^{-c_{1}},\left(g_{3}^{-c_{3}}, g_{2}^{-c_{2}}\right)\right)\left(g_{3}^{-c_{3}}, g_{2}^{-c_{2}}\right)^{-1} \\
& \quad\left(g_{3}^{-c_{3}}, g_{2}^{-c_{2}-1}\right)\left(g_{1}^{-c_{1}},\left(g_{3}^{-c_{3}}, g_{2}^{-c_{2}-1}\right)\right)^{-1}\left(g_{2}^{-c_{2}-1}, g_{1}^{-c_{1}}\right)^{-1} .
\end{aligned}
$$

General case. We start by describing a maximal tree in $E=E_{m}$. We construct this tree inductively as a union of lines. For the two-dimensional case, denote the coordinates in $\mathbb{R}^{2}$ by $\left(y_{1}, y_{m}\right)$. We take as a maximal tree in $E=E_{2}$ the union of the lines $\left\{y_{1}=C_{1}\right\}$ with $C_{1} \in \mathbb{Z}$ and the line $\left\{y_{m}=0\right\}$. Now assume that a maximal tree is chosen in $E_{k+1} \subset \mathbb{R}^{k+1}$ and describe how to chose such a tree in $E_{k+2} \subset \mathbb{R}^{k+2}$. Denote the coordinates in $\mathbb{R}^{k+1}$ by $\left(y_{1}, \ldots, y_{k}, y_{m}\right), k<m-1$. The maximal tree in $E_{k+1}$ constructed on the previous step is a union of $(k+1)$ families of lines $\left\{Q_{1}\right\}, \ldots,\left\{Q_{k+1}\right\}$. Then we pass to $\mathbb{R}^{k+2}$ with coordinates $\left(y_{1}, \ldots, y_{k+1}, y_{m}\right)$ and take as a maximal tree in $E_{k+2}$ the union of the following families of lines:

$$
\begin{aligned}
& \left\{Q_{1} ; y_{k+1}=C_{k+1}\right\}, \quad\left\{Q_{2} ; y_{k+1}=C_{k+1}\right\}, \ldots, \\
& \left\{Q_{k+1} ; y_{k+1}=C_{k+1}\right\}, \quad\left\{y_{1}=\cdots=y_{k}=y_{m}=0\right\} .
\end{aligned}
$$

Note that we appended each family from the previous step by an additional equation $y_{k+1}=C_{k+1}, C_{k+1} \in \mathbb{Z}$, and added one more family consisting of a single line $\left\{y_{1}=\cdots=y_{k}=y_{m}=0\right\}$. At the end we obtain in $E=E_{m}$ a maximal tree which a union of $m$ families of lines. After contracting this maximal tree we obtain a wedge of circles $\widehat{E}$. The circles in this wedge correspond bijectively to those edges of the graph $E$ that do not belong to the contracted maximal tree. These edges of $E$ are segments of the form

$$
I=\left[\left(c_{1}, \ldots, c_{m}\right),\left(c_{1}, \ldots, c_{p}+1, \ldots, c_{m}\right)\right], \quad c_{i} \in \mathbb{Z}, p \in\{1, \ldots, m-1\} .
$$

where $c_{i} \in \mathbb{Z}$. Every such segment can be completed to a loop in $E$ by adding edges from the contracted maximal tree, and we need to express the word corresponding to this loop via the commutators (3.1).

First consider a segment (3.3) with $p \neq m-1$. This segment $I$ corresponds to a loop in $E$ consisting of $I$ and segments from the contracted maximal tree, given by the word

$$
\chi=g_{m-1}^{c_{m-1}} g_{1}^{c_{1}} g_{2}^{c_{2}} \cdots g_{m-2}^{c_{m-2}} g_{m}^{c_{m}} g_{p} g_{m}^{-c_{m}} g_{m-2}^{-c_{m-2}} \cdots g_{p}^{-c_{p}-1} \cdots g_{1}^{-c_{1}} g_{m-1}^{-c_{m-1}} .
$$

Let $\widehat{I}$ be the orthogonal projection of $I$ onto the hyperplane $y_{m-1}=0$. The loop corresponding to $\widehat{I}$ can be expressed via the commutators (3.1) not containing $g_{m-1}$, by the induction assumption. Let this expression be given by a word $\psi$. 
Then $\chi=\left(g_{m-1}^{-c_{m-1}}, \psi\right) \cdot \psi^{-1}$. The commutator $\left(g_{m-1}^{-c_{m-1}}, \psi\right)$ can also be expressed via the commutators (3.1). To do this we use the identity

$$
\begin{array}{r}
\left(g_{q}^{n_{q}},\left(g_{p}^{n_{p}}, x\right)\right)=\left(g_{q}^{n_{q}}, x\right)\left(x,\left(g_{p}^{n_{p}}, g_{q}^{n_{q}}\right)\right)\left(g_{q}^{n_{q}}, g_{p}^{n_{p}}\right)\left(x, g_{p}^{n_{p}}\right)\left(g_{p}^{n_{p}},\left(g_{q}^{n_{q}}, x\right)\right) \\
\left(x, g_{q}^{n_{q}}\right)\left(g_{p}^{n_{p}}, g_{q}^{n_{q}}\right)\left(g_{p}^{n_{p}}, x\right) .
\end{array}
$$

This identity allows us to swap the element $g_{m-1}^{-c_{m-1}}$ with other elements $g_{i}^{-c_{i}}$ in iterated commutators until $g_{m-1}^{-c_{m-1}}$ reaches the position prescribed by the ordering of indices in (3.1). This argument is the same as in [PV] Lemma 4.7], and more details can be found there.

Now consider a segment (3.3) with $p=m-1$, that is,

$$
I=\left[\left(c_{1}, \ldots, c_{m}\right),\left(c_{1}, \ldots, c_{m-1}+1, c_{m}\right)\right] .
$$

The corresponding loop in $E$ is given by the word

$$
\begin{aligned}
\chi=g_{m-1}^{c_{m-1}} g_{1}^{c_{1}} g_{2}^{c_{2}} & \ldots g_{m-2}^{c_{m-2}} g_{m}^{c_{m}} g_{m-1} g_{m}^{-c_{m}} g_{m-2}^{-c_{m-2}} \ldots g_{1}^{-c_{1}} g_{m-1}^{-c_{m-1}-1}= \\
& =g_{m-1}^{c_{m-1}} \psi g_{m-1} \xi g_{m-1}^{-c_{m-1}-1}=\left(g_{m-1}^{-c_{m-1}}, \psi\right) \psi^{-1}\left(g_{m-1}^{-c_{m-1}-1}, \xi\right) \xi^{-1},
\end{aligned}
$$

where $\psi$ and $\xi$ do not contain $g_{m-1}$. The words $\psi$ and $\xi$ can be expressed via the commutators (3.1) not containing $g_{m-1}$, by the induction assumption. The rest of the argument is the same as in the previous paragraph.

We have therefore proved that any element of the commutator subgroup $F_{m}^{\prime}$ can be expressed via the iterated commutators (3.1). It remains to prove that the system of generators (3.1) is free. Let $I^{m}(s)$ be a cube in $\mathbb{R}^{m}$ with edges of length $s \in \mathbb{Z}$ and vertices in the lattice $\mathbb{Z}^{m}$. Let $E_{m}^{(s)}:=E_{m} \cap I^{m}(s)$. Then $E_{m}^{(s)}$ is a finite graph, and $G_{m}^{(s)}=\pi_{1}\left(E_{m}^{(s)}\right)$ is a free group of finite rank. We can position the cubes $I^{m}(s), s=1,2, \ldots$, in such a way that they include in each other and $\mathbb{R}^{m}=\bigcup_{s=1}^{\infty} I^{m}(s)$. The free group $F_{m}^{\prime}=\pi_{1}\left(E_{m}\right)$ of infinite rank is the inductive limit of the groups $G_{m}^{(s)}$. It is enough to prove that each group $G_{m}^{(s)}$ is freely generated by the iterated commutators (3.1) corresponding to loops inside the cube $I^{m}(s)$. For simplicity, assume that $I^{m}(s)$ is the cube in the positive orthant of $\mathbb{R}^{m}$ with a vertex at the origin. Then a commutator of the form (3.1) corresponds to a loop in $I^{m}(s)$ if and only if all exponents of the elements in the commutator satisfy $0<n_{k} \leqslant s$. The number of commutators (3.1) with exponents in this range is given by

$$
J_{m}^{(s)}=\sum_{i=2}^{m}\left(\begin{array}{c}
m \\
i
\end{array}\right)(i-1) s^{i}
$$

Denote by $W_{m}^{(s)}$ the rank of the free group $G_{m}^{(s)}$. It is equal to the number of circles in the wedge obtained by contracting a maximal tree in the graph $E_{m}^{(s)}$. We need to show that $J_{m}^{(s)}=W_{m}^{(s)}$.

By considering a maximal tree in the graph $E_{2}^{(s)}$ we obtain $W_{2}^{(s)}=s^{2}$. Furthermore, we have a recursive relation

$$
W_{m}^{(s)}=W_{m-1}^{(s)}(s+1)+(s+1)^{m-1} s-s .
$$

It implies that

$$
W_{m}^{(s)}=W_{m-l}^{(s)}(s+1)^{l}+l(s+1)^{m-1} s-s \sum_{i=0}^{l-1}(s+1)^{i},
$$


for $0 \leqslant l \leqslant m-2$. In particular, for $l=m-2$ we obtain

$$
W_{m}^{(s)}=s^{2}(s+1)^{m-2}+(m-2)(s+1)^{m-1} s-s \sum_{i=0}^{m-3}(s+1)^{i} .
$$

Since both $J_{m}^{(s)}$ and $W_{m}^{(s)}$ are polynomials in $s$, we only need to compare their coefficients. Calculating the coefficient of $s^{k}$ in (3.6) we obtain

$$
\begin{aligned}
\left(\begin{array}{c}
m-2 \\
k-2
\end{array}\right)+(m-2) & \left(\begin{array}{c}
m-1 \\
k-1
\end{array}\right)-\sum_{i=k-1}^{m-3}\left(\begin{array}{c}
i \\
k-1
\end{array}\right) \\
=\left(\begin{array}{c}
m-2 \\
k-2
\end{array}\right)+(m-2) & \left(\begin{array}{c}
m-1 \\
k-1
\end{array}\right)-\left(\begin{array}{c}
m-2 \\
k
\end{array}\right) \\
& =m\left(\begin{array}{c}
m-1 \\
k-1
\end{array}\right)-\left(\begin{array}{c}
m \\
k
\end{array}\right)=(k-1)\left(\begin{array}{c}
m \\
k
\end{array}\right) .
\end{aligned}
$$

This is precisely the coefficient of $s^{k}$ in $J_{m}^{(s)}$, see (3.5).

\section{Generators for the commutator subgroup of a right-Angled Artin} GROUP

Theorem 4.1. Let $R A_{\mathcal{K}}$ be the right-angled Artin group corresponding to a simplicial complex $\mathcal{K}$ on $m$ vertices. The commutator subgroup $R A_{\mathcal{K}}^{\prime}$ has a minimal generator set consisting of iterated commutators

$$
\left(g_{j}^{n_{j}}, g_{i}^{n_{i}}\right),\left(g_{k_{1}}^{n_{k_{1}}},\left(g_{j}^{n_{j}}, g_{i}^{n_{i}}\right)\right), \ldots,\left(g_{k_{1}}^{n_{k_{1}}},\left(g_{k_{2}}^{n_{k_{2}}}, \ldots,\left(g_{k_{m-2}}^{n_{k_{m}-2}},\left(g_{j}^{n_{j}}, g_{i}^{n_{i}}\right)\right) \ldots\right)\right)
$$

where $n_{k} \in \mathbb{Z} \backslash\{0\}, k_{1}<k_{2}<\cdots<k_{m-2}<j>i, k_{s} \neq i$ for any $s$, and $i$ is the smallest vertex in a connected component not containing $j$ of the subcomplex $\mathcal{K}_{\left\{k_{1}, \ldots, k_{\ell-2}, j, i\right\}} \cdot$

Proof. In the case when $\mathcal{K}$ consists of $m$ disjoint points the statement is proved in Lemma 3.1. Adding an edge $\{p, q\}$ to the complex $\mathcal{K}$ results in adding the commutation relation $\left(g_{p}, g_{q}\right)=1$ to the right-angled Artin group. We shall eliminate commutators from the set (3.1) which do not appear in (4.1) using the new commutation relations. This argument is similar to the corresponding argument in the case of a right-angled Coxeter group, see [PV, Theorem 4.6], and we only outline the main steps.

First assume that the vertices $j$ and $i$ are in the same connected component of the complex $\mathcal{K}_{\left\{k_{1}, \ldots, k_{\ell-2}, j, i\right\}}$. We shall show that the corresponding commutator $\left(g_{k_{1}}^{n_{k_{1}}},\left(g_{k_{2}}^{n_{k_{2}}}, \ldots,\left(g_{k_{\ell-2}}^{n_{k_{\ell-2}}},\left(g_{j}^{n_{j}}, g_{i}^{n_{i}}\right)\right) \ldots\right)\right)$ can be excluded from the generating set. We choose a path from $i$ to $j$, that is, choose vertices $i_{1}, \ldots, i_{q}$ from $k_{1}, \ldots, k_{\ell-2}$ with the property that $\mathcal{K}$ contains the edges $\left\{i, i_{1}\right\},\left\{i_{1}, i_{2}\right\}, \ldots,\left\{i_{q-1}, i_{q}\right\},\left\{i_{q}, j\right\}$. We proceed by induction on the length of the path. Induction starts from the commutator $\left(g_{j}^{n_{j}}, g_{i}^{n_{i}}\right)=1$ corresponding to a one-edge path $\{i, j\} \in \mathcal{K}$. Now assume that the path consists of $q+1$ edges. Using the relation (3.4) we can move the elements $g_{i_{1}}^{n_{i_{1}}}, g_{i_{2}}^{n_{i_{2}}}, \ldots, g_{i_{q}}^{n_{i_{q}}}$ in $\left(g_{k_{1}}^{n_{k_{1}}},\left(g_{k_{2}}^{n_{k_{2}}}, \cdots\left(g_{k_{\ell-2}}^{n_{k_{\ell-2}}},\left(g_{j}^{n_{j}}, g_{i}^{n_{i}}\right)\right) \cdots\right)\right)$ to the right and restrict ourselves to the commutator $\left(g_{i_{1}}^{n_{i_{1}}},\left(g_{i_{2}}^{n_{i_{2}}}, \cdots\left(g_{i_{q}}^{n_{i_{q}}},\left(g_{j}^{n_{j}}, g_{i}^{n_{i}}\right)\right) \cdots\right)\right)$. Using (3.4) together with the commutation relation $\left(g_{i_{q}}^{n_{i_{q}}}, g_{j}^{n_{j}}\right)=1$ coming from the edge $\left\{i_{q}, j\right\} \in \mathcal{K}$ we convert the commutator $\left(g_{i_{1}}^{n_{i_{1}}},\left(g_{i_{2}}^{n_{i_{2}}}, \cdots\left(g_{i_{q}}^{n_{i_{q}}},\left(g_{j}^{n_{j}}, g_{i}^{n_{i}}\right)\right) \cdots\right)\right)$ to the commutator $\left(g_{j}^{n_{j}},\left(g_{i_{1}}^{n_{i_{1}}}, \cdots\left(g_{i_{q-1}}^{n_{i_{q-1}}},\left(g_{i_{q}}^{n_{i_{q}}}, g_{i}^{n_{i}}\right)\right) \cdots\right)\right)$ modulo commutators of shorter length. The latter contains the commutator $\left(g_{i_{1}}^{n_{i_{1}}}, \cdots\left(g_{i_{q-1}}^{n_{i_{q-1}}},\left(g_{i_{q}}^{n_{i_{q}}}, g_{i}^{n_{i}}\right)\right) \cdots\right)$ corresponding to a shorter path $\left\{i, i_{1}, \ldots, i_{q}\right\}$. By the inductive hypothesis it can be 
expressed through commutators of shorter length and therefore excluded from the set of generators.

We therefore obtain a generator set for $R A_{\mathcal{K}}^{\prime}$ consisting of nested commutators $\left(g_{k_{1}}^{n_{k_{1}}}, \cdots\left(g_{k_{\ell-2}}^{n_{k_{\ell-2}}},\left(g_{j}^{n_{j}}, g_{i}^{n_{i}}\right)\right) \cdots\right)$ with $j$ and $i$ in different connected components of $\mathcal{K}_{\left\{k_{1}, \ldots, k_{\ell-2}, j, i\right\}}$. Consider commutators $\left(g_{k_{1}}^{n_{k_{1}}}, \cdots\left(g_{k_{\ell-2}}^{n_{k_{\ell-2}}},\left(g_{j}^{n_{j}}, g_{i_{1}}^{n_{i_{1}}}\right)\right) \cdots\right)$ and $\left(g_{k_{1}^{\prime}}^{n_{k_{1}^{\prime}}}, \cdots\left(g_{k_{\ell-2}^{\prime}}^{n_{k_{\ell-2}^{\prime}}},\left(g_{j}^{n_{j}}, g_{i_{2}}^{n_{i_{2}}}\right)\right) \cdots\right)$ with the property that $\left\{k_{1}, \ldots, k_{\ell-2}, j, i_{1}\right\}=$ $\left\{k_{1}^{\prime}, \ldots, k_{\ell-2}^{\prime}, j, i_{2}\right\}$ and $i_{1}, i_{2}$ lie in the same connected component of $\mathcal{K}_{\left\{k_{1}, \ldots, k_{\ell-2}, j, i_{1}\right\}}$ which is different from the connected component containing $j$. We claim that one of these commutators can be expressed through the other and commutators of shorter length. To see this, we argue as in the previous paragraph, i. e. we consider a path between $i_{1}$ and $i_{2}$ in $\mathcal{K}_{\left\{k_{1}, \ldots, k_{\ell-2}, j, i_{1}\right\}}$ and then reduce it inductively to a one-edge path. This leaves us with the pair of commutators $\left(g_{i_{2}}^{n_{i_{2}}},\left(g_{j}^{n_{j}}, g_{i_{1}}^{n_{i_{1}}}\right)\right)$ and $\left(g_{i_{1}}^{n_{i_{1}}},\left(g_{j}^{n_{j}}, g_{i_{2}}^{n_{i_{2}}}\right)\right)$, where $\left\{i_{1}, i_{2}\right\} \in \mathcal{K},\left\{i_{1}, j\right\} \notin \mathcal{K},\left\{i_{2}, j\right\} \notin \mathcal{K}$. The claim then follows easily from the relation $\left(g_{i_{1}}^{n_{i_{1}}}, g_{i_{2}}^{n_{i_{2}}}\right)=1$.

Now, to enumerate independent commutators, we use the convention of writ$\operatorname{ing}\left(g_{k_{1}}^{n_{k_{1}}}, \cdots\left(g_{k_{\ell-2}}^{n_{k_{\ell-2}}},\left(g_{j}^{n_{j}}, g_{i}^{n_{i}}\right)\right) \cdots\right)$ where $i$ is the smallest vertex in its connected component within $\mathcal{K}_{\left\{k_{1}, \ldots, k_{\ell-2}, j, i\right\}}$. This leaves us with precisely the set of commutators (4.1).

It remains to show that the generating set (4.1) is minimal. To do this we use the "exhausting" procedure as in the final part of the proof of Theorem 3.1. Recall that $R A_{\mathcal{K}}^{\prime}=\pi_{1}\left(\mathcal{L}_{\mathcal{K}}\right)$, see Theorem 2.4 Let $I^{m}(s)$ be a lattice cube with edges of length $s$. Set $\mathcal{L}_{\mathcal{K}}^{(s)}=\mathcal{L}_{\mathcal{K}} \cap I^{m}(s)$. Then $\mathcal{L}_{\mathcal{K}}^{(s)}$ is a finite cell complex (a cubic complex) and $G_{m}^{(s)}=\pi_{1}\left(\mathcal{L}_{\mathcal{K}}^{(s)}\right)$ is a finitely generated group. We can assume $\mathbb{R}^{m}=\bigcup_{s \in \mathbb{N}} I^{m}(s)$, so that $\mathcal{L}_{\mathcal{K}}=\bigcup_{s \in \mathbb{N}} \mathcal{L}_{\mathcal{K}}^{(s)}$. The infinitely generated group $R A_{\mathcal{K}}^{\prime}=\pi_{1}\left(\mathcal{L}_{\mathcal{K}}\right)$ is the inductive limit of the groups $G_{m}^{(s)}$. It is enough to prove that the commutators (4.1) corresponding to the loops inside $I^{m}(s)$ give a minimal generating set for the group $G_{m}^{(s)}$. The fact that these commutators generate $G_{m}^{(s)}$ follows from the argument above; we only need to check the minimality.

A commutator of the form (4.1) corresponds to a loop in $I^{m}(s)$ if and only if all exponents of the elements in the commutator satisfy $0<n_{k} \leqslant s$. The number of commutators (4.1) with exponents in this range is given by

$$
P_{m}^{(s)}=\sum_{J \subset[m]}\left(\operatorname{cc}\left(\mathcal{K}_{J}\right)-1\right) s^{|J|},
$$

where $\operatorname{cc}\left(\mathcal{K}_{J}\right)$ is the number of connected components of the complex $\mathcal{K}_{J}$, and $|J|$ denotes the cardinality of the set $J$. Consider the first integer homology group $H_{1}\left(\mathcal{L}_{\mathcal{K}}^{(s)}\right)$. Since $G_{m}^{(s)}=\pi_{1}\left(\mathcal{L}_{\mathcal{K}}^{(s)}\right)$, we have $H_{1}\left(\mathcal{L}_{\mathcal{K}}^{(s)}\right)=G_{m}^{(s)} /\left(G_{m}^{(s)}\right)^{\prime}$. On the other hand, the space $\mathcal{L}_{\mathcal{K}}^{(s)}$ is a polyhedral product of the form $\left(I_{s}, Z_{s+1}\right)^{\mathcal{K}}$, where $I_{s}=$ $[0, s]$ is the segment of length $s$ and $Z_{s+1}=\{0, \ldots, s\}$ is the set integer point on this segment. Then Theorem 2.5 implies that

$$
H_{1}\left(\mathcal{L}_{\mathcal{K}}^{(s)}\right) \cong \widetilde{H}_{0}\left(\bigvee_{J \subset[m]}\left|\mathcal{K}_{J}\right| \wedge Z_{s+1}^{\wedge J}\right) \cong \widetilde{H}_{0}\left(\bigvee_{J \subset[m]}\left|\mathcal{K}_{J}\right|^{\vee\left(s^{|J|}\right)}\right)
$$

Comparing this with (4.2) we obtain that $H_{1}\left(\mathcal{L}_{\mathcal{K}}^{(s)}\right)$ is a free abelian group of $\operatorname{rank} P_{m}^{(s)}$ (because $\widetilde{H}_{0}(X \vee Y)=\widetilde{H}_{0}(X) \oplus \widetilde{H}_{0}(Y)$ and $\left.\operatorname{rank} \widetilde{H}_{0}\left(\left|\mathcal{K}_{J}\right|\right)=\operatorname{cc}\left(\mathcal{K}_{J}\right)-1\right)$. Therefore, the number of generators of the group $G_{m}^{(s)}=\pi_{1}\left(\mathcal{L}_{\mathcal{K}}^{(s)}\right)$ is at least $P_{m}^{(s)}$, as needed. 
According to a result of of Servatius, Droms and Servatius [SDS], the commutator subgroup $R A_{\mathcal{K}}^{\prime}$ is free if and only if the 1 -skeleton $\mathcal{K}^{1}$ is a chordal graph (see also [PV] Corollary 4.4]). A graph without loops and double edges is called chordal (or triangulated) if each of its cycles with $\geqslant 4$ vertices has a chord (an edge joining two vertices that are not adjacent in the cycle). When $\mathcal{K}^{1}$ is a chordal graph, Theorem 4.1 gives a basis for the free group $R A_{\mathcal{K}}^{\prime}$.

\section{Example 4.2.}

1. Let $\mathcal{K}$ be a 5 -cycle, shown in Figure 1 , left. For the corresponding right-angled
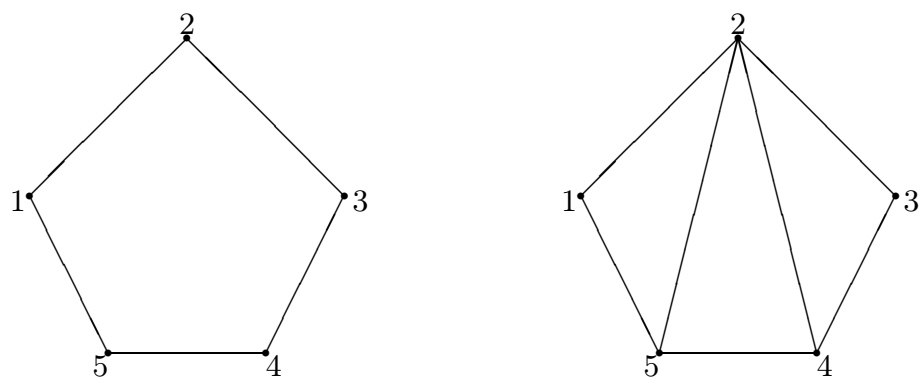

Figure 1. A 5-cycle (left) and a chordal graph (right).

Artin group $R A_{\mathcal{K}}$, Theorem 4.1 gives a generator set for the commutator subgroup $R A_{\mathcal{K}}^{\prime}$ consisting of the commutators

$$
\left(g_{3}^{n_{3}}, g_{1}^{n_{1}}\right),\left(g_{4}^{n_{4}}, g_{1}^{n_{1}}\right),\left(g_{4}^{n_{4}}, g_{2}^{n_{2}}\right),\left(g_{5}^{n_{5}}, g_{2}^{n_{2}}\right),\left(g_{5}^{n_{5}}, g_{3}^{n_{3}}\right),
$$

$\left(g_{4}^{n_{4}},\left(g_{5}^{n_{5}}, g_{2}^{n_{2}}\right)\right),\left(g_{3}^{n_{3}},\left(g_{5}^{n_{5}}, g_{2}^{n_{2}}\right)\right),\left(g_{1}^{n_{1}},\left(g_{5}^{n_{5}}, g_{3}^{n_{3}}\right),\left(g_{3}^{n_{3}},\left(g_{4}^{n_{4}}, g_{1}^{n_{1}}\right),\left(g_{2}^{n_{2}},\left(g_{4}^{n_{4}}, g_{1}^{n_{1}}\right)\right.\right.\right.$

with $n_{k} \in \mathbb{Z} \backslash\{0\}$. The graph $\mathcal{K}^{1}$ is not chordal, so the group $R A_{\mathcal{K}}^{\prime}$ is not free. In fact, $R A_{\mathcal{K}}^{\prime}$ contains a surface group of genus 5 , generated by the ten commutators above with all exponents $n_{i}=1$, which satisfy a single relation. This surface group is the commutator subgroup for the right-angled Coxeter group corresponding to $\mathcal{K}$, see [PV, Example 4.8].

2. Now let $\mathcal{K}$ be the 1-dimensional simplicial complex (graph) shown in Figure 1 , right. This graph is chordal, so the corresponding group $R A_{\mathcal{K}}^{\prime}$ is free. Theorem 4.1 gives a basis consisting of the commutators

$$
\left(g_{3}^{n_{3}}, g_{1}^{n_{1}}\right),\left(g_{4}^{n_{4}}, g_{1}^{n_{1}}\right),\left(g_{5}^{n_{5}}, g_{3}^{n_{3}}\right),\left(g_{1}^{n_{1}},\left(g_{5}^{n_{5}}, g_{3}^{n_{3}}\right),\left(g_{3}^{n_{3}},\left(g_{4}^{n_{4}}, g_{1}^{n_{1}}\right)\right.\right.
$$

with $n_{k} \in \mathbb{Z} \backslash\{0\}$.

\section{Generalisation to Graph PRODUCts}

Definition 5.1. Let $\mathcal{K}$ be a simplicial complex on $[m]$ and let $\boldsymbol{G}=\left(G_{1}, \ldots, G_{m}\right)$ be a sequence of $m$ groups. The graph product $G^{\mathcal{K}}$ is defined as the quotient group

$$
G^{\mathcal{K}} \cong \underset{k=1}{\star} G_{k} /\left(g_{i} g_{j}=g_{j} g_{i} \text { for } g_{i} \in G_{i}, g_{j} \in G_{j},\{i, j\} \in \mathcal{K}\right),
$$

where $\star_{k=1}^{m} G_{k}=G_{1} \star \cdots \star G_{m}$ denotes the free product of the groups $G_{k}$.

The graph product $G^{\mathcal{K}}$ depends only on the 1 -skeleton of $\mathcal{K}$. There is also a categorical definition of the graph product $G^{\mathcal{K}}$ as a colimit of the groups $G^{I}=$ $\prod_{i \in I} G_{i}$ over the category of faces $I \in \mathcal{K}$, see [PV] Construction 2.5] for the details. When each $G_{i}$ is $\mathbb{Z}$, the graph product $G^{\mathcal{K}}$ is the right-angled Artin group $R A_{\mathcal{K}}$.

Remark. Although graph products can be defined for (well-behaved) topological groups [PRV], we only consider discrete groups $G_{i}$ here. 
There is a canonical epimorphism $G^{\mathcal{K}} \rightarrow \prod_{k=1}^{m} G_{k}$ obtained by letting $g_{i} \in G_{i}$ and $g_{j} \in G_{j}$ commute for any pair $\{i, j\}$. When each group $G_{k}$ is abelian, the group $\operatorname{Ker}\left(\boldsymbol{G}^{\mathcal{K}} \rightarrow \prod_{k=1}^{m} G_{k}\right)$ coincides with the commutator subgroup $\left(\boldsymbol{G}^{\mathcal{K}}\right)^{\prime}$.

Theorem 5.2. Let $\mathcal{K}$ be a flag simplicial complex on $m$ vertices, let $\boldsymbol{G}=$ $\left(G_{1}, \ldots, G_{m}\right)$ be a sequence of $m$ nontrivial groups, and let $G^{\mathcal{K}}$ be the corresponding graph product group.

(a) $\operatorname{Ker}\left(\boldsymbol{G}^{\mathcal{K}} \rightarrow \prod_{k=1}^{m} G_{k}\right)$ is a free group if and only if $\mathcal{K}^{1}$ is a chordal graph;

(b) $\operatorname{Ker}\left(G^{\mathcal{K}} \rightarrow \prod_{k=1}^{m} G_{k}\right)$ has a minimal generator set consisting of iterated commutators

$$
\left(g_{j}, g_{i}\right),\left(g_{k_{1}},\left(g_{j}, g_{i}\right)\right), \ldots,\left(g_{k_{1}},\left(g_{k_{2}}, \ldots,\left(g_{k_{m-2}},\left(g_{j}, g_{i}\right)\right) \ldots\right)\right),
$$

where $g_{k} \in G_{k} \backslash\{1\}, k_{1}<k_{2}<\cdots<k_{m-2}<j>i, k_{s} \neq i$ for any $s$ and $i$ is the smallest vertex in a connected component not containing $j$ of the subcomplex $\mathcal{K}_{\left\{k_{1}, \ldots, k_{\ell-2}, j, i\right\}}$.

Part (a) is proved in [PV, Theorem 4.3]. This result may look surprising, as the condition in the criterion depends only on the combinatorics of $\mathcal{K}$ and does not depend on the group structure of particular $G_{k}$. In particular, when $\mathcal{K}$ is $m$ disjoint points, we obtain that

$$
K_{m}:=\operatorname{Ker}\left(G_{1} \star \cdots \star G_{m} \rightarrow G_{1} \times \cdots \times G_{m}\right)
$$

is a free group. The reason is that $\operatorname{Ker}\left(\boldsymbol{G}^{\mathcal{K}} \rightarrow \prod_{k=1}^{m} G_{k}\right)$ is the fundamental group of the polyhedral product $(E \boldsymbol{G}, \boldsymbol{G})^{\mathcal{K}}$, and therefore it depends only on the cardinality of $G_{i}$ (as the groups are discrete), and does not depend on their group structure.

The proof of (b) follows the same line as for Theorem 4.1. We give a sketch emphasising the most crucial points; a more detailed proof is included in the $\mathrm{PhD}$ thesis of the second author.

Proof of Theorem 5.2 (b). First, we consider the group $K_{m}$ corresponding to the case when $\mathcal{K}$ is $m$ disjoint points. Observe that $K_{2}=\operatorname{Ker}\left(G_{1} \star G_{2} \rightarrow G_{1} \times G_{2}\right)$ is generated by the commutators $\left(g_{2}, g_{1}\right)$ with $g_{i} \in G_{i} \backslash\{1\}$. Indeed, a word $g_{i_{1}} g_{j_{1}} g_{i_{2}} \cdots g_{i_{p}} g_{j_{p}} \in G_{1} \star G_{2}$ with $g_{i_{k}} \in G_{1}, g_{j_{k}} \in G_{2}, k=1, \ldots, m$, belongs to $K_{2}$ if and only if $g_{i_{1}} \cdots g_{i_{p}}=1$ in $G_{1}$ and $g_{j_{1}} \cdots g_{j_{p}}=1$ in $G_{2}$. Now we split off commutators from the word in the same way as in the case $m=2$ of Theorem 3.1. In the case of $K_{m}$ we argue by induction as follows. Write an element of $K_{m}$ as $g_{i_{1}} h_{j_{1}} g_{i_{2}} \cdots g_{i_{p}} h_{j_{p}}$ with $g_{i_{k}} \in G_{m}, h_{j_{k}} \in G_{1} \star \cdots \star G_{m-1}$ and $g_{i_{1}} \cdots g_{i_{p}}=1$ in $G_{m}$. Then split off commutators from the left as

$$
\begin{aligned}
g_{i_{1}} h_{j_{1}} g_{i_{2}} \cdots g_{i_{p}} h_{j_{p}}=\left(g_{i_{1}}^{-1}, h_{j_{1}}^{-1}\right) h_{j_{1}} g_{i_{1}} g_{i_{2}} \cdots g_{i_{p}} h_{j_{p}} \\
=\left(g_{i_{1}}^{-1}, h_{j_{1}}^{-1}\right)\left(h_{j_{1}}^{-1},\left(g_{i_{1}} g_{i_{2}}\right)^{-1}\right) \cdots g_{i_{p}} h_{j_{p}}=\ldots
\end{aligned}
$$

until we end up at a product of the form $c_{1} \cdots c_{q} h^{\prime}$ where each $c_{i}$ is a commutator of the form $(g, h)$ or $(h, g)$ with $g \in G_{m}, h \in G_{1} \star \cdots \star G_{m-1}$, and $h^{\prime} \in K_{m-1}$. Then we write $h^{\prime}$ in a similar way using the inductive assumption. Now we use identities like $(a b, c)=(a, c)((a, c), b)(b, c)$ to show that $K_{m}$ is generated by iterated commutators $\left(g_{1}, \ldots, g_{p}\right)$ of length $p \geqslant 2$ with arbitrary bracketing and $g_{i} \in G_{\alpha_{i}}$.

The next step is to express an iterated commutator $\left(g_{1}, \ldots, g_{p}\right)$ with arbitrary bracketing in terms of canonical nested commutators $\left(g_{1}, \cdots\left(g_{p-2},\left(g_{p-1}, g_{p}\right)\right) \cdots\right)$; this is done is the standard way using the commutator identities as in [Wa] or [MKS]. Then we use the commutator identity similar to (3.4):

$$
\left(g_{q},\left(g_{p}, x\right)\right)=\left(g_{q}, x\right)\left(x,\left(g_{p}, g_{q}\right)\right)\left(g_{q}, g_{p}\right)\left(x, g_{p}\right)\left(g_{p},\left(g_{q}, x\right)\right)\left(x, g_{q}\right)\left(g_{p}, g_{q}\right)\left(g_{p}, x\right)
$$

(here $g_{p}$ is an arbitrary element of $G_{\alpha_{p}}$, while in (3.4) it denoted a generator of $\left.G_{\alpha_{p}} \cong \mathbb{Z}\right)$. It allows us to swap elements in a canonical nested commutator until they 
reach the positions prescribed by the ordering of indices in 3.1). This shows that the group $K_{m}$ is generated by precisely the commutators described in Theorem5.2 (b).

To show that the described commutators generate the group $G(\mathcal{K}):=\operatorname{Ker}\left(\boldsymbol{G}^{\mathcal{K}} \rightarrow\right.$ $\left.\prod_{k=1}^{m} G_{k}\right)$ for arbitrary $\mathcal{K}$ we argue exactly as in the proof of Theorem 4.1 eliminate commutators not appearing in the generator set using the new commutation relations in $G^{\mathcal{K}}$ and the commutator identity from the previous paragraph.

When establishing the minimality of the generator set, we replace $\mathcal{L}_{\mathcal{K}}=(\mathbb{R}, \mathbb{Z})^{\mathcal{K}}$ by the polyhedral product $(E \boldsymbol{G}, \boldsymbol{G})^{\mathcal{K}}$, which is the classifying space for the group $G(\mathcal{K})$ by [St, Theorem 1.1] or [PV], Theorem 3.2]. First assume that each $G_{k}$ is a finite group. Then the number of commutators in Theorem 5.2 (b) is finite and given by

$$
P_{m}=\sum_{J \subset[m]}\left(\left(\operatorname{cc}\left(\mathcal{K}_{J}\right)-1\right) \prod_{j \in J}\left(\left|G_{j}\right|-1\right)\right),
$$

where $\operatorname{cc}\left(\mathcal{K}_{J}\right)$ is the number of connected components of the complex $\mathcal{K}_{J}$, and $\left|G_{j}\right|$ denotes the cardinality of $G_{j}$. On the other hand, Theorem 2.5] implies that

$$
\begin{aligned}
G(\mathcal{K}) / G^{\prime}(\mathcal{K})=H_{1} & \left((E \boldsymbol{G}, \boldsymbol{G})^{\mathcal{K}}\right) \\
& \cong \widetilde{H}_{0}\left(\bigvee_{J \subset[m]}\left|\mathcal{K}_{J}\right| \wedge \boldsymbol{G}^{\wedge J}\right) \cong \widetilde{H}_{0}\left(\bigvee_{J \subset[m]}\left|\mathcal{K}_{J}\right|^{\vee \prod_{j \in J}\left(\left|G_{j}\right|-1\right)}\right) .
\end{aligned}
$$

Hence, $G(\mathcal{K}) / G^{\prime}(\mathcal{K})$ is a free abelian group of rank $P_{m}$. Therefore, the number of generators of the group $G(\mathcal{K})$ is at least $P_{m}$, as needed.

In the case when each $G_{k}$ is countable infinite we can use the "exhausting" procedure as in Theorem 4.1. Note that the group-theoretic properties of $G_{k}$ do not affect this argument; only the cardinalities matter.

In the case of arbitrary (discrete) $G_{k}$, if the generator set is not minimal, then some iterated commutator $\left(g_{1}, \ldots, g_{p}\right)$ from the given generator set can be eliminated by expressing it through other commutators in the generator set. Denote by $H_{k} \subset G_{k}$ the subgroup generated by all elements of $G_{k}$ entering this expression. Then the same expression gives a relation in the graph product group $\boldsymbol{H}^{\mathcal{K}}$, showing that the generator set is also not minimal for $\boldsymbol{H}^{\mathcal{K}}$. However, each group in the sequence $\boldsymbol{H}=\left(H_{1}, \ldots, H_{m}\right)$ is finitely generated and therefore countable, which is a contradiction.

\section{REFERENCES}

[BBCG] Bahri, Anthony; Bendersky, Martin; Cohen, Frederic R.; Gitler, Samuel. The polyhedral product functor: a method of computation for moment-angle complexes, arrangements and related spaces. Adv. Math. 225 (2010), no. 3, 1634-1668.

[BK] Bokut', Leonid A.; Kukin, Georgy P. Algorithmic and Combinatorial Algebra. Mathematics and its Applications, 255. Kluwer Academic Publishers Group, Dordrecht, 1994.

[BP1] Buchstaber Victor; Panov, Taras. Torus actions, combinatorial topology and homological algebra. Uspekhi Mat. Nauk 55 (2000), no. 5, 3-106 (Russian). Russian Math. Surveys 55 (2000), no. 5, 825-921 (English).

[BP2] Buchstaber, Victor; Panov, Taras. Toric Topology. Math. Surv. and Monogr., 204, Amer. Math. Soc., Providence, RI, 2015.

[BEMPP] Buchstaber, Victor; Erokhovets, Nikolay; Masuda, Mikiya; Panov, Taras; Park, Seonjeong. Cohomological rigidity of manifolds defined by right-angled 3-dimensional polytopes. Uspekhi Mat. Nauk 72 (2017), no. 2, 3-66 (Russian); Russian Math. Surveys 72 (2017), no. 2, 199-256 (English translation); arXiv:1610.07575.

[HW] Hurley, T. C.; Ward, M. A. Bases for commutator subgroups of a free group. Proc. Roy. Irish Acad. Sect. A 96 (1996), no. 1, 43-65.

[KR] Kim, Ki Hang; Roush, Fred W. Homology of certain algebras defined by graphs. J. Pure Appl. Algebra 17 (1980), no. 2, 179-186.

[MKS] Magnus, Wilhelm; Karrass, Abraham; Solitar, Donald. Combinatorial group theory. Presentations of groups in terms of generators and relations. Second revised edition. Dover Publications, Inc., New York, 1976. 
[PRV] Panov, Taras; Ray, Nigel; Vogt, Rainer. Colimits, Stanley-Reiner algebras, and loop spaces. In: Categorical decomposition techniques in algebraic topology (Isle of Skye, 2001). Progress in Math., 215, Birkhäuser, Basel, 2004, pp. 261-291.

[PV] Panov, Taras; Veryovkin, Yakov. Polyhedral products and commutator subgroups of rightangled Artin and Coxeter groups. Mat. Sbornik 207 (2016), no. 11, 105-126 (Russian); Sbornik Math. 207 (2016), no. 11, 1582-1600 (English translation); arXiv:1603.06902

[SDS] Herman Servatius, Carl Droms and Brigitte Servatius. Surface subgroups of graph groups. Proc. Amer. Math. Soc. 106 (1989), no. 3, 573-578.

[St] Stafa, Mentor. On the fundamental group of certain polyhedral products. J. Pure Appl. Algebra 219 (2015), no. 6, 2279-2299.

[Wa] Waldinger, Hermann. The lower central series of groups of a special class. J. Algebra 14 (1970), no. 2, 229-244.

Department of Mathematics and Mechanics, Moscow State University, Leninskie Gory, 119991 Moscow, Russia,

Institute for Theoretical and Experimental Physics, Moscow, Russia and Institute for Information Transmission Problems, Russian Academy of Sciences E-mail address: tpanov@mech.math.msu.su $U R L:$ http://higeom.math.msu.su/people/taras/

Steklov Mathematical Institute, Russian Academy of Sciences E-mail address: verevkin_j.a@mail.ru 